\title{
Handover in VLC Systems with Cooperating Mobile Devices*
}

\author{
Anna Maria Vegni ${ }^{\S}$, and Thomas D.C. Little ${ }^{\dagger}$ \\ $\S$ Department of Applied Electronics, \\ University of Roma Tre, Rome, Italy \\ amvegni@uniroma3.it \\ ${ }^{\dagger}$ Department of Electrical and Computer Engineering, \\ Boston University, Boston MA, USA \\ tdcl@bu.edu
}

October 18, 2011

MCL Technical Report No. 10-06-2011

\begin{abstract}
Visible Light Communications (VLC) is emerging as viable means to overcome the crowded radio spectrum for highly-localized communications systems. With such an approach, luminaires can be adopted for supporting data communication in addition to illumination in what we call Cellular Lighting. However, in these systems, the directionality of light as the communication medium introduces new challenges in sustaining network connectivity especially under mobility scenarios.

In this paper we investigate mobility in VLC devices and propose an approach to managing handover adopted from wireless cellular systems, but with very small cells and directional characteristic. Moreover, this technique provides the coordination and cooperation among VLC devices to achieve seamless connectivity in two different indoor scenarios consisting of overlapping (uniform lighting) and non-overlapping (spotlighting) cases. Results indicate how different design decisions in supporting luminaire placement achieve full coverage at varying data rates.
\end{abstract}

*In Proc. of Mobility and Communication for Cooperation and Coordination (MC3) workshop, part of the IEEE Intl. Conference on Computing, Networking and Communications (ICNC 2012), January 30 - February 2, 2012, Maui, Hawaii, USA. This work is supported, in part, by the Engineering Research Centers Program of the National Science Foundation under NSF Cooperative Agreement No. EEC-0812056. Any opinions, findings and conclusions or recommendations expressed in this material are those of the author(s) and do not necessarily reflect those of the National Science Foundation. 


\section{Introduction}

In the next generation of wireless communication technologies, the development of new Laser Diodes (LD) and Light-Emitting Diode (LED) materials are driving researchers to Free Space Optical (FSO) solutions as a viable and promising technology alternative to the use of the radio spectrum (RF) [1]. Indeed, FSO systems enable the use for short-range indoor applications in addition to previous outdoor long-range cases.

Visible Light Communications (VLC) is an emerging optical wireless communication technology in which baseband signals are modulated on the light emitted by typically white LEDs [2]. The use of LED-based lighting technology is exploited to assess an opportunistic deployment of widespread FSO communications. LEDbased network transceivers have a variety of competitive advantages over RF, such as low cost, small size, energy savings and high bandwidth density. As a result, these unique advantages have the potential to motivate widespread adoption of this nearfield communications technology whenever luminaries are introduced for lighting. The net is the creation of lighting that comprises wireless coverage using the lighting field, or Cellular Lighting.

Compared to traditional wireless communication technologies, the VLC physical medium is unregulated by the FCC (US) and has no identified health risks (i.e., no risks distinct from that of current lighting systems). The features of directivity (Line-of-Sight or LOS) and high obstacle impermeability provide secure data transmission within a closed indoor environment. Leveraging these attributes, VLC technology is highly applicable to indoor environments where new energyefficient LED materials and devices will replace old incandescent and fluorescent lights. However, FSO communications become more difficult unless terminals hold fixed positions (i.e. in front of a desk, sitting on a chair, etc.). Mobile communications via VLC in an indoor environment are challenging due to occlusions that can occur due to orientation or physical obstructions in an indoor volume [3].

In this paper we present a feasibility analysis of mobility in VLC systems, and propose a handover (HO) mechanism for seamless connectivity when mobile devices are moving inside an indoor environment comprised of a set of "white" LED-based lighting cells. The proposed HO technique can work both in reactive and proactive modes, aiming to limit network disconnections. This aspect is also provided through the cooperation of mobile devices in the environment, which share data information of lighting cells.

The organization of the paper is as follows; in Section 2 we describe the main aspects of a VLC system and briefly introduce our prototypes [4]. In Section 3 we give an overview of handover mechanism, as typically applied in cellular systems. In Section 4 we introduce the mobility issue in VLC systems, and formalize the problem of seamless connectivity in an indoor environment covered by a set of LED-based lighting cells. Subsections 4.1 and 4.2 deal with two typical use cases i.e., the separated and overlapping lighting cells scenarios, respectively. In Section 5 we show the data rate distribution in a LED-based Cellular-Lighting-scenario, validated via simulation results performed with Candles modeling software [5]. Finally, conclusions are drawn in Section 6.

\section{VLC systems}

The LED-based visible-light optical medium is becoming an attractive substitute for RF communication. This vision has been largely demonstrated and proven in the research community [6, 7]. 


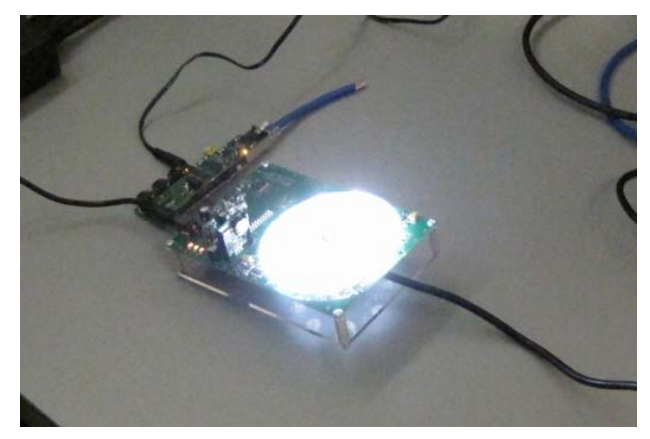

Figure 1: Prototype VLC Transceiver using White LEDs.

At Boston University we tested a short range (ceiling height $3 \mathrm{~m}$ ) duplex point-to-point white-LED system with a data rate of $2 \mathrm{Mb} / \mathrm{s}$; $40 \mathrm{SL} 1$ prototypes were developed, describing typical scenarios associated with future access points providing LED-based indoor networking [4, 8]. The SL1 prototype has a duplex channel characteristic of one-half of an asymmetric hybrid RF-FSO model, as shown in Figure 1. Data can be downloaded at high speed from overhead illumination to a receiver, for example, to support data streaming for high definition video to a laptop. The obtained results are very promising for providing multiple-access for ubiquitous indoor networking. Using off-the-shelf LEDs and photodiodes we can demonstrate viable communication using visible light in the presence of normal room illumination, and in the presence of matching transceivers used as lighting configured in an adjacent array with spacing of $0.75 \mathrm{~m}$.

This prototype represents a static scenario, in which end-to-end data transmission is LOS between a pair of devices in fixed positions. More challenging is the situation in which one device is mobile; introducing discontinuities in LOS communications the service maintenance is an issue.

\section{Handover Mechanism}

In this section we investigate recent works on seamless connectivity in Optical Wireless (OW) systems. In next generation networks the use of heterogeneous technologies relies on the growing user demand to be seamlessly connected to the Internet, maintaining high Quality-of-Service (QoS) services, as well as avoiding network congestions and delays.

In mobility scenarios users can maintain their connectivity through an automatic and seamless mechanism, called as handover $(\mathrm{HO})$ [9]. This represents a switching process from one serving network to a candidate network. Typical handover is known as horizontal handover which allows connectivity switching whenever a mobile terminal moves from an access point to another without changing the service access network [9]. HO was initially adopted in cellular system to avoid service disconnections due to mobility issues. An HO is traditionally initiated on the basis of physical parameter measurements, such as the Received Signal Strength (RSS), providing the service maintenance when the power level from a serving cell decreases. Optimality criteria for handover decisions balance different factors including, monetary cost, energy consumption, positioning and enduser QoS $[10,11]$.

$\mathrm{RF}$ and $\mathrm{OW}$ access technologies have been investigated as hybrid systems achieving seamless 
connectivity [12, 13]. In [12], a fuzzy logic-based vertical handover decision algorithm is proposed for an integrated WiFi-IR system. The benefits of both IR and WiFi schemes achieve optimal handover when handover decisions consider short- and long-period interruptions. Moreover, in [14] the authors investigate the potential of a combination of VLC and WiFi for an indoor broadcasting service.

In this paper we investigate how basic horizontal handover mechanisms can be applied in VLC systems in which the user connectivity switches from one lighting cell to another. Uniform and spotlighting coverage have been assumed in this work. By exploiting the analogy between the RSS parameter in cellular systems and the Received Signal Intensity (RSI) in optical wireless, we extend the traditional RSS-based handover procedures into an RSI-based technique for mobile VLC systems.

\section{Mobility in VLC systems}

VLC systems can be distinguished on the basis of their lighting modes [15], such as the use of $(i)$ uniform lighting coverage, and (ii) spotlighting. The uniform lighting mode attempts a total coverage by distributing wide Field-of-View (FOV) LED chips uniformly on the ceiling. In this case, the FOVs from all the LEDs overlap each other, resulting in an essentially continuous lighting field. In contrast, spotlighting VLC produces intense and focused light, often used to enhance the uniform lighting scheme. Spotlighting offers multiple benefits, including higher data rate densities and reduced channel distortion. Spotlighting is mainly used to light small areas, such as for reading lights in airplane passenger seats, and illumination of desktops in cubicle offices or libraries. We consider both uniform and spotlighting modes; we also introduce a hybrid scheme as a combination of both lighting modes to provide wide area data coverage. An example of hybrid scheme considers desk lamps (spotlighting) providing bright lighting on work surfaces, while overhead lighting (uniform) covers other areas where lower light intensity levels are sufficient, such as hallways and aisles.

A lighting cell typically has a very small coverage with respect to other wireless systems or even femtocells. However, we design the lighting cell radius on the basis of transmitted data rate. Our LED-based lighting device (luminaire) is assumed to produce an "ideal" cone of light, i.e. its entire light output is projected as a circular lighting field with a hard boundary. The "ideal" cone can be approximated by means of an ellipsoidal reflector, so that to achieve a required signal irradiance at the receiver side $p_{R x}\left[\mathrm{~W} / \mathrm{m}^{2}\right]$, the needed transmitted light power $P_{T x}[\mathrm{~W}]$ is $P_{T x}=\pi r^{2} p_{R x}$ with $r$ the light field radius at the receiver plane.

The design of lighting cell size is based on the following assumptions: (i) a Mobile Terminal (MT) crosses a lighting cell at a constant speed, $v[\mathrm{~m} / \mathrm{s}]$, (ii) each cell has an omnidirectional lighting coverage with radius $r$ [m], and (iii) each LED Access Point (AP) transmits at a nominal data rate in the centre of the cell, $B$ [b/s]. We assume that an MT is equipped with a VLC network interface card so that it receives data when is inside a lighting cell. We are now able to define the following concept of cell gain. Given a mobile terminal crossing a lighting cell at constant speed $v[\mathrm{~m} / \mathrm{s}]$, the cell gain [bits] is the number of bits received by the mobile terminal during the time interval it spends while traversing crossing the lighting cell. It is defined as:

$$
G_{\text {cell }} \leq \tilde{B} \cdot 2 t_{r}
$$

where $\tilde{B}[\mathrm{~b} / \mathrm{s}]$ is the effective data rate of the lighting cell, and $t_{r}[\mathrm{~s}]$ is the time necessary for 


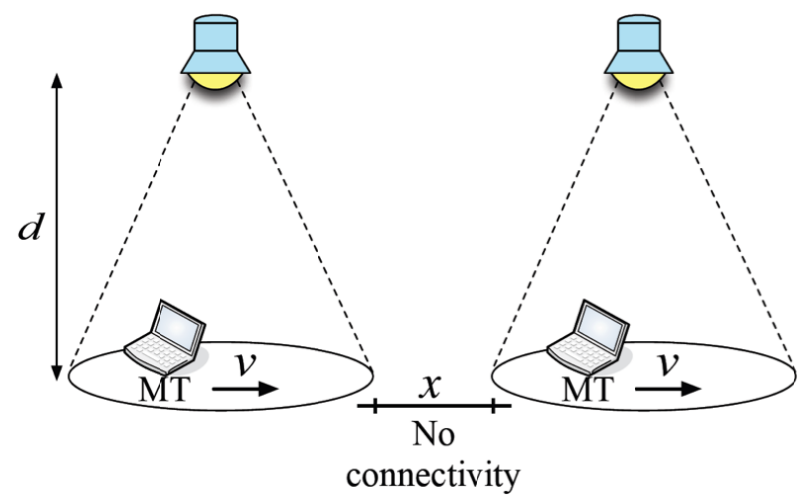

(a)

connectivity

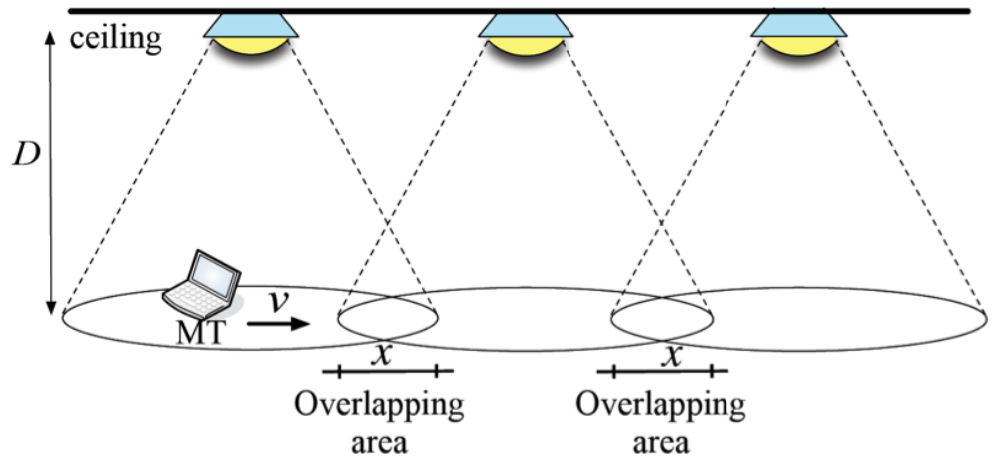

(b)

Figure 2: (a) Non-overlapping spotlighting, and (b) overlapping uniform lighting cells scenario, respectively.

walking a distance $r[\mathrm{~m}]$ at speed $v[\mathrm{~m} / \mathrm{s}]$ inside the cell (i.e., $\left.t_{r}=r / v\right)$. Notice that the effective data rate is expressed as a percentage of the nominal data rate $B[\mathrm{~b} / \mathrm{s}]$. From (1), the geometry of a lighting cell (i.e., the cell radius) is $r \geq G_{\text {cell }} v / 2 \tilde{B}$ which shows the strict dependance of the radius on the user QoS requirements, expressed in terms of cell gain. Leveraging this consideration, we can state that the lighting cell size increases for high QoS requirements, providing high data rate, while it decreases for low QoS requirements at low data rates. For instance, let us assume a MT is moving at speed $v$ (i.e., $v=1 \mathrm{~m} / \mathrm{s}$ ) inside a serving lighting cell with radius $r$. The MT is requiring a low QoS service provision at data rate $b$ (i.e., $b=10 \mathrm{~kb} / \mathrm{s}$ ), and the cell gain is $G_{c e l l}=10 \mathrm{~kb}$. According to (1), it follows that $r=50 \mathrm{~cm}$. Notice that the smaller the radius, the smaller the number of LEDs achieving a desired irradiance. For example, an irradiance corresponding to $2\left[\mathrm{~W} / \mathrm{m}^{2}\right]$ on an area of radius $r=50 \mathrm{~cm}$ can be achieved by a LED source to output around $1.57 \mathrm{~W}$ of light, corresponding to approximately $353 \mathrm{~lx}$ from a $353 \mathrm{~lm}$ source, for typical white LEDs.

After defining the cell gain, we introduce the following indoor scenarios: $(i)$ a separated spotlighting scenario at distance $x[\mathrm{~m}]$ (see Figure $2(a)$ ), and (ii) an overlapping uniform lighting cell scenario, at overlapping size in the range $0<x<r[\mathrm{~m}]$ (see Figure $2(b)$ ). 


\subsection{Scenario with separated spotlighting cells}

In this case, achieving seamless connectivity is a problem. The scenario is comprised of a set of LED-based spotlighting cells, separated by a gap of distance $x[\mathrm{~m}]$, as depicted in Figure $2(a)$. Here there is no overlapping coverage area. The transmitters are each located at a distance $d$ from the plane of the receivers, e.g., ceiling to desktop, each projecting a light field of radius $r$ on the receiver plane. The receiver FOVs are assumed to be wide throughout.

A MT moves on a straight direction at speed $v[\mathrm{~m} / \mathrm{s}]$ and approaches the borderline of a LEDbased Serving Lighting Cell (SLC). When the MT exits the lighting coverage, the gap between two consecutive spotlights affects the ongoing connectivity, and service is interrupted. The MT should find a new spotlighting cell after walking a distance greater than $x[\mathrm{~m}]$. During the time interval $t_{x} \geq x / v[\mathrm{~s}]$, the MT has no connectivity.

To avoid connectivity interruptions, one solution relies on increasing the buffer size at the MT, upon leaving the SLC, and storing an estimated quantity of bits, i.e., $G=\tilde{B} \cdot(x / v)$, that is the minimum amount of bits the MT would lose on walking $x$ meters before finding a New Lighting Cell (NLC).

This approach provides the handover execution in a reactive manner, that means the MT initiates a handover procedure before breaking the connection with the SLC. The MT can know if it will find a new neighboring spotlighting cell before exiting the SLC, due to a simple intensity test. When the MT approaches the boundary of the SLC, it will check for the presence of other spotlights through any Received Signal Intensity (RSI) levels $\left[\mathrm{W} / \mathrm{m}^{2}\right]$. The intensity test checks the presence of overlapping cells, and works as first alarm whenever the RSI level from the SLC decreases below a fixed threshold. This alarm is negative if there are neighboring overlapping lighting cells; it is positive if no overlapping lighting cells are available. Only in the latter case, the buffer size at the MT will increase in order to store the amount of bit $G$. Notice that this solution recalls the compressed-mode used for inter-system and interfrequency hard handover in UMTS [16].

\subsection{Scenario with overlapping uniform lighting cells}

In the second case, the overlapping uniform lighting cells will avoid disconnections if an HO is promptly adopted. Figure $2(b)$ shows the considered scenario. Let us assume that three uniform lighting cells have equal radius $r[\mathrm{~m}]$, and nominal data rate $B[\mathrm{~b} / \mathrm{s}]$. The cells overlap in pairs forming a lighting coverage i.e., $0<x<r[\mathrm{~m}]$, regardless of distance $D$ (i.e., $d<D$ ) from the plane of the receiver. A MT moves in straight direction inside the SLC at speed $v[\mathrm{~m} / \mathrm{s}]$, receiving an effective data rate $\tilde{B}[\mathrm{~b} / \mathrm{s}]$. When the MT crosses the lighting coverage $x[\mathrm{~m}]$, she will be connected at an increasing data rate, respectively from the uniform SLC and a neighboring NLC. In this case the intensity test will be negative and a proactive handover will be initiated; the ongoing connection will be seamlessly switched to the NLC during the time interval $t_{x}$. To guarantee a constant QoS level, the cell gain parameter should be the same for all the uniform lighting cells the MT will cross. In the whole overlapping area the gain parameter is:

$$
G=n \tilde{B} \cdot(x / v)
$$

where $n$ is the number of overlapping uniform lighting cells in the same area (e.g., 2).

Notice that mobile devices moving inside the indoor environment can cooperate and share the 
information of positions of lighting cells, in order to prevent disconnections. In this way an handover will be reactive.

\section{Data Rate Distribution}

In this section we describe the data rate distribution in a VLC system comprised of LED-based lighting cells (Cellular Lighting). This analysis yields which handover mechanism to adopt reactive or proactive - for different lighting coverage. Simulation results show the handover algorithm is affected by access point positioning, generating different data rate coverage.

In our prototypes, we use "white" LUXEON Rebel LEDs, whose features are ultra-compact, surface-mount, and high-power device [17]. LEDs are produced with semiconductor technology, which is subject to process variation. This aspect can yield a range of flux performance that is approximately Gaussian in nature. According to typical representative spatial radiation pattern for Cool, Neutral and Warm White Lambertian [17], the data rate profile $B[\mathrm{~b} / \mathrm{s}]$ in each lighting cell can be approximated with a Gaussian distribution, i.e., $\mathcal{N}\left(\mu, \sigma^{2}\right)$ where $\mu$ is the mean value corresponding to the centre of the lighting cell, and $\sigma$ is the standard deviation, i.e., $2 \sigma=2(r-\varepsilon)$, with $\varepsilon$ as the portion of the radius $r$ where the RSI level has an intensity reduction.

Figure 3 (a) depicts the analytical behavior of data rate distribution for three APs, respectively placed in positions $P_{1}=(2,2,3), P_{2}=(4,4,3)$, and $P_{3}=(6,6,3)$, aligned each other along the same line. Each AP shows the same Gaussian distribution of the data rate, for $\sigma=2$, and $B=1 \mathrm{Mb} / \mathrm{s}$. As expected, the three data rate distributions overlap each other, and the handover mechanism will relay in the overlapping uniform lighting cells scenario. Notice the effective data rate in the center of the lighting cells is $<0.2 \mathrm{Mb} / \mathrm{s}$, as well as validated via simulation results in Figure $3(b)$.

The same configuration has been analyzed for $\sigma=1$, as depicted in Figure 3(c) and $(d)$. In this case the data rate distributions shows a narrow trend and the effective data rate is increased, while the overlapping area between neighboring lighting cells is smaller than in Figure $3(a)$ and $(b)$. This is mainly due to a decrease of the standard deviation (i.e., $\sigma=1$ ). Notice that on opportunistically modifying the standard deviation, we can approximate the uniform and spotlighting behavior (i.e., low values of standard deviations are for spotlighting, while high values are for uniform lighting coverage). The smaller the standard deviation the narrower the FOV, and the lower the data rate reduction in the center of the cell.

Finally, we analyzed a typical indoor scenario depicting a conference room, with a $2 \mathrm{D} n \times m$ array of lighting cells. No walls or obstacles nearby to cause light reflection or diffraction were assumed in the room. Through the knowledge of data rate distribution inside the conference room, the handover mechanism will be reactive, driven on the basis of the presence of distance gaps between neighboring lighting cells. In contrast, in the areas with overlapping data rate distributions the handover will be proactive. The size of the simulated conference room is $10 \times 10$ meters, with a set of $3 \times 3$ LED-based lighting cell array, as shown in Figure $4(a)$. Three APs in positions $(5,2,3),(5,5,3)$, and $(5,8,3)$ depict a spotlighting behavior with $B=10 \mathrm{Mb} / \mathrm{s}$ and $\sigma=1$. All the other APs are uniform lighting devices, with $B=50 \mathrm{Mb} / \mathrm{s}$ and $\sigma=4$. We considered different values of data rate in order to provide a real hybrid scheme (i.e., spotlighting plus uniform lighting coverage). The analytical profile of data rate distribution inside the conference room is shown in Figure $4(b)$. The APs are near each other and the lighting cells overlap, forming indistinct huge 


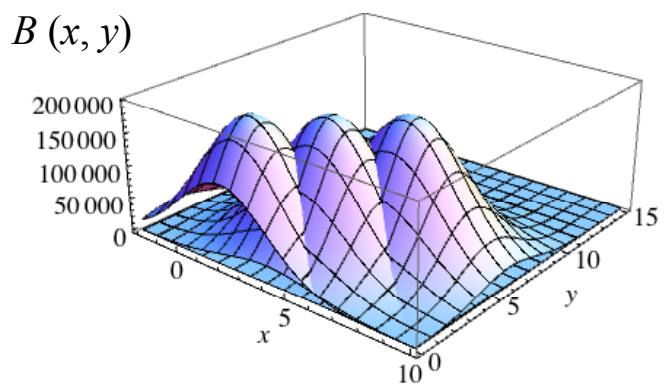

(a)

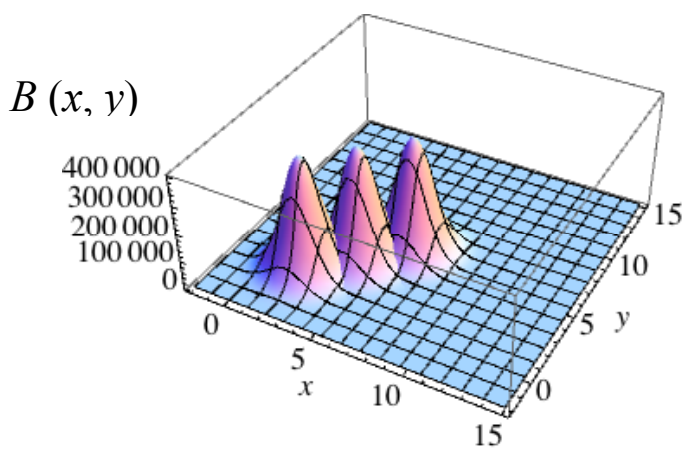

(c)

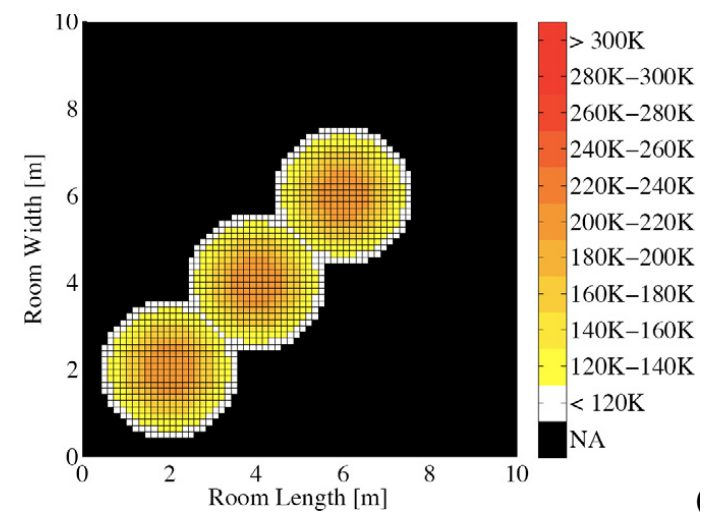

(b)

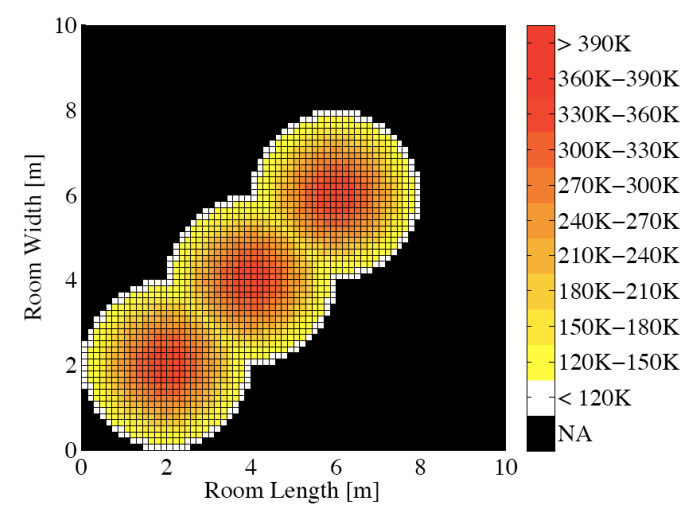

(d)

Figure 3: Data rate distribution for three LED APs in an indoor overlapping cell scenario. (a) Analytical trend for $\sigma=2$, and $(b)$ simulated results via Candles software [5]. (c) Analytical trend for $\sigma=1$, and $(d)$ simulated results via Candles software [5].

FOVs, whose peaks of data rates are over $14 \mathrm{Mb} / \mathrm{s}$, as proven via simulation in Figure $4(c)$. Notice that the spotlighting cells are completely covered by uniform lighting. As a result, the whole room achieves a $71.8 \%$ coverage with data rate higher than $5 \mathrm{Mb} / \mathrm{s}$, in the range $[14.3,0.93] \mathrm{Mb} / \mathrm{s}$. The handover mechanism will be proactive in almost the whole area with overlapping lighting cells.

\section{Conclusions}

In this paper we have analyzed the mobility issues in indoor VLC systems comprised of Cellular Lighting. The seamless connectivity is achieved through an RSI-based handover mechanism working in both reactive and proactive modes. Two typical use-case scenarios have been investigated, with uniform overlapping and separated spotlighting cells, respectively; a hybrid scenario has also been considered.

Simulation results validate the analytical trend, and shown the handover algorithm is able to adapt in different lighting coverage configurations. Future work will consider prototyping of the 


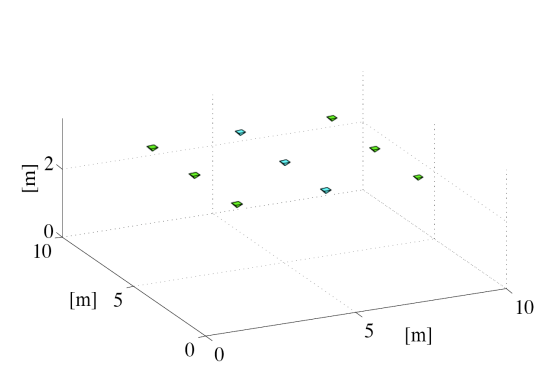

(a)

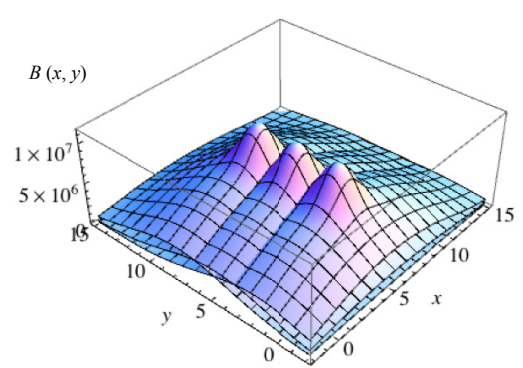

(b)

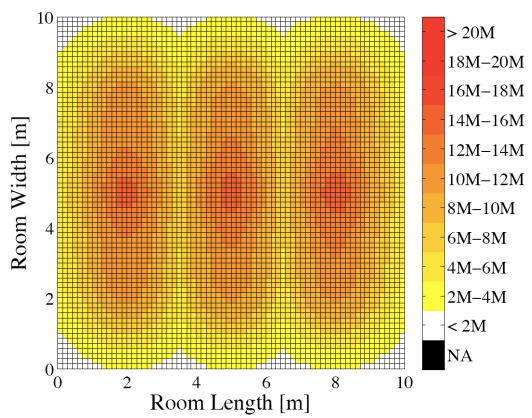

(c)

Figure 4: (a) Geometry of the conference room with nine "white" LED access points. (b) Analytical behavior of data rate distribution for spotlighting $(\sigma=1)$, and uniform lighting cells $(\sigma=4)$.

(c) Data rate simulation results via Candles software [5].

concepts in a lighting testbed to demonstrate the proposed handover technique.

\section{References}

[1] A. Boucouvalas. "Challenges in Optical Wireless Communications". Optical Photonic News, 16(9):36-39, 2005.

[2] J.B. Carruthers. Wireless Infrared Communications. Wiley Encyclopedia of Telecommunications, 2002.

[3] J. Hou and D.C. O'Brien. "Polling scheme for Indoor LOS Optical Wireless LAN". Electron. Lett., 39(10):794-795, 2003.

[4] Z. Wu and T.D.C. Little. "Network Solutions for the LOS Problem of New Indoor Free Space Optical System". In IEEE, IEE Int. Symp. on Communications Systems, Networks, and Digital Signal Processing, Newcastle, UK, July 2010.

[5] M. Rahaim, T. Borogovac, and J.B. Carruthers. "Candles: Communications and lighting emulation software". In Proc. on 5th ACM Int. Workshop on Wireless Network Testbeds, Experimental Evaluation and Characterization, 2010.

[6] http://www.vlcc.net.

[7] T. Douseki. “A Batteryless Optical-wireless System with White-LED Illumination”. In Proc. on 15th IEEE International Symposium on Personal, Indoor and Mobile Radio Communications, 2004.

[8] T.D.C. Little, P. Dib, K. Shah, N. Barraford, and B. Gallagher. "Using LED Lighting for Ubiquitous Indoor Wireless Networking". In Proc. of 4th IEEE International Conference on Wireless and Mobile Computing, Networking and Communications, Avignon, France, October 2008. 
[9] G.P. Pollini. “Trends in handover design”. IEEE Communications Magazine, 34(3):82-90, 1996.

[10] M. Kassar, B. Kervella, and G. Pujolle. "An Overview of Vertical Handover Decision Strategies in Heterogeneous Wireless Networks”. Computer Communications, 31(10), 2008.

[11] T. Inzerilli, A.M. Vegni, A. Neri, and R. Cusani. "A Cross-Layer Location-Based Approach for Mobile-Controlled Connectivity". International Journal of Digital Multimedia Broadcasting, 2010:13, 2010.

[12] J. Hou and D.C. O’Brien. "Vertical Handover Decision-Making Algorithm using Fuzzy Logic for the Integrated Radio-and-OW system". IEEE Trans. on Wireless Communications, 5(1):176-185, January 2006.

[13] D.J.T. Heatley, D.R. Wisely, I. Neild, and P. Cochrane. "Optical wireless: the story so far". IEEE Communications Magazine, 36(12):72-74, 79-82, December 1998.

[14] J. Rufo et al. "Experimental Evaluation of Video Transmission through LED Illumination Devices". IEEE Trans. on Consumer Electronics, 56(3):1411-1416, August 2010.

[15] T. Borogovac, M. Rahaim, and J. Carruthers. "Spotlighting for Visible Light Communications and Illumination". In Proc. of 1st IEEE Workshop on Optical Wireless Communications, IEEE GLOBECOM, 2010.

[16] H. Holma and A. Toskala. WCDMA for UMTS, Radio Access for Third Generation Mobile Communications. Wiley \& sons, Ltd., 3rd edition, 2004.

[17] Philips Lumileds Lighting Company. LUXEON Rebel Datasheet DS56 (09/03/24). 